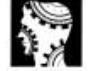

original papers offenders. Our respondents clearly appreciated being consulted, and expressed a wish for further training and support not just for the patients directly but for themselves in dealing with the vast bulk of psychological distress that stays within primary care.

\section{Declaration of interest}

None.

\section{Acknowledgements}

We thank Dr Jane Fryer (Medical Director, Southwark Primary Care Trust) for her support of this project, and for the large number of Southwark GPs who took the time to respond; Drs Emma Janes and Herbert Steiner for help in the earlier part of this project; Ms Elaine Allsworth for her painstaking data entry; Drs Marta Buszewicz and Lana Koro-Kondza for permission to adapt and use their questionnaire.

\section{References}

ANGERMEYER, M. C. \& MATSCHINGER, BATEMAN, A. \& FONAGY, P. H. (1996) Public attitude towards psychiatric treatment. Acta Psychiatrica Scandanavia, 94, 326-336. (1999) Effectiveness of partia hospitalization in the treatment of borderline personality disorder. American Journal of Psychiatry, 156 1563-1569. 2006) Public beliefs about and attitudes towards people with mental illness: a review of population studies. Acta Psychiatrica Scandanavia, 113, $163-179$

APPLEBY, L. (2004) The National Service Framework for Mental Health - Five Years On. Department of Health.

BASS, C., PEVELER, R. \& HOUSE, R. (2001) Somatoform disorders: severe psychiatric illnesses neglected by psychiatrists. British Journal of Psychiatry, 179, 11-14.

CENTRE FOR ECONOMIC PERFORMANCE'S MENTAL HEALTH POLICY GROUP (2006) The Depression
Report: A New Deal for Depression and Anxiety Disorders. London School of Economics.

CLERKIN, J. F., LEVY, K. N. LENZENWEGER, M. F. et al (2007)

Evaluating three treatments for psychotherapy for borderline personality disorder: a multiwave study. American Journal of Psychiatry, 164, 922-928.

DEPARTMENT OF HEALTH (1999)

National Service Framework for Mental Health: Modern Standards and Service Models. Department of Health (http:// www.dh.gov.uk/en/Publicationsand statistics/Publications/

PublicationsPolicyAndGuidance/ DH_4009598.

\section{DEPARTMENT OF HEALTH (2001)}

Treatment Choice in Psychological

Therapies and Counselling: Evidence Based Practice Guideline. Department of Health (http://www.dh.gov.uk/en/ Publicationsandstatistics/

Publications/PublicationsPolicyAnd Guidance/DH_4007323).

DEPARTMENT OF HEALTH (2004)

Organising and Delivering

PsychologicalTherapies. Department of Health (http://www.dh.gov.uk/en/ Publicationsandstatistics/

Publications/PublicationsPolicyAnd Guidance/DH_4086100)

\section{DEPARTMENT OF HEALTH (2007)} Improving Access to Psychological Therapies: Commissioning a Brighter Future. Department of Health (http://

www.dh.gov.uk/en/Publicationsand statistics/Publications/Publications PolicyAndGuidance/DH.074556).

GABBARD, G. (2007) Do all roads lead to Rome? New findings on borderline personality disorder [editorial]. American Journal of Psychiatry, 164, $853-855$

GIESEN-BLOO, J., VAN DYCK, R SPINHOVEN, P., et al (2006) Outpatient psychotherapy for borderline personality disorder. Archives of General Psychiatry, 63, 649-658.

MORAN, P., RENDU, A., JENKINS, R et al (2001) The impact of personality disorder in UK primary care: a 1-year follow-up of attenders. Psychological Medicine, 31, 1447-1454.

MORAN, P. \& MANN, A. (2002) The prevalence and 1 -year outcome of cluster B personality disorders in primary care. Journal of Forensic Psychiatry, 13, 527-537.

OLDHAM, J. (2007) Psychodynamic psychotherapy for borderline personality disorder [editorial]. American Journal of Psychiatry, 164, 1465-1467.

ROYAL COLLEGE OF PSYCHIATRISTS (2001) Curriculum for Basic Specialist Training and the MRCPsych Examination (CR95). Royal College of Psychiatrists.

ROYAL COLLEGE OF GENERAL PRACTITIONERS (2005) Information Sheet No. 22: Stress and Genera Practice. Royal College of General Practitioners.

*Anne Ward Consultant Psychiatrist in Psychotherapy, Maudsley Psychotherapy Service, OPD Maudsley Hospital, Denmark Hill, London SE5 8AZ, email: Anne.Ward@slam.nhs.uk, Giovanni Polizzi Consultant Psychiatrist in Psychotherapy, Maudsley Psychotherapy Service, OPD Maudsley Hospital, Miomir Milovanovic Consultant Psychiatrist in Psychotherapy, SouthWest London \& St George's NHS Trust, Psychotherapy Department, Springfield Hospital

STEVE ONYETT, KAREN LINDE， GYLES GLOVER， SIOBHAN FLOYD， STEVEN BRADLEY AND HUGH MIDDLETON

\title{
Implementation of crisis resolution/home treatment teams in England: national survey 2005-2006
}

\section{AIMS AND METHOD}

To describe implementation of crisis resolution/home treatment (CRHT) teams in England, examine obstacles to implementation and priorities for development. We conducted an online survey followed by a telephone or face-to-face interview among 243 teams.

TSee original paper pp. 378-379, this issue.
RESULTS

Considerable progress has been made in implementation with a subset of teams demonstrating strong fidelity to the Department of Health's guidance, particularly in urban settings. However, only $40 \%$ of teams described themselves as fully established. Many teams reported a high assessment load, understaffing, limited multidisciplinary input and patchy fulfilment of their gatekeeping role.

\section{CLINICAL IMPLICATIONS}

Successful implementation of the CRHT teams as alternatives to hospital admission requires resources for home treatment out of hours, effective systems working among local services, stronger local understanding and advocacy of the teams' role. 
Crisis resolution/home treatment (CRHT) teams have been central to English mental health policy since 1999. Their implementation has been rigorously performancemanaged. The Department of Heath (2000) set a target of 100000 people being served by 335 CRHT teams by December 2005. The teams aim to provide an alternative to hospital admission by intervening in the pathway between community-based referrers and in-patient care, providing robust assessment and gate-keeping of admissions. This requires a $24-h$ service to users in their own homes and opportunities to resolve crises in the contexts in which they occur.

Outcomes of CRHT team intervention include reduced length of stay (Johnson et al, 2005), reduced rates of admission where teams provide out-of-hours cover (Johnson et al, 2005; Glover et al, 2006), costeffectiveness (Joy et al, 2001), high satisfaction among users and families (Dean et al, 1993; Joy et al, 2001; Johnson et al, 2005), and better staff morale (Minghella et al, 1998).

\section{Method}

The implementation of CRHT teams, perceived obstacles and priorities for development were surveyed between late 2005 and early 2006.

A comprehensive questionnaire was developed through national networks of CRHT providers, piloting with ten services, and formatted for online data entry. Using secure access, respondents were asked to complete the questionnaire in preparation for a telephone or face-to-face interview. The research team supported internet access and provided telephone assistance to respondents completing the online questionnaire.

Teams were included in the study if they had been designed to achieve the outcomes required of a CRHT team locally as described in the Mental Health Policy Implementation Guide (Department of Health, 2002). We identified 243 such teams by drawing together information from the national database then held at the University of Durham and local intelligence provided by Care Service Improvement Partnership CRHT leads.

\section{Analysis}

Quantitative data were analysed using non-parametric statistics (SPSS version 13 for Windows) because they were either categorical measures or were not normally distributed. Kruskal-Wallis $\chi^{2}$ or the $\chi^{2}$ coefficient was used depending on the number of categories explored. Post hoc multiple comparisons employed the MannWhitney $U$-test for associations between interval level variables and chi-squared test for associations between two-level categorical data. The Spearman's rank correlation coefficient $\left(r_{\mathrm{s}}\right)$ was used to examine relationships between interval level variables. Free-text material was collected in response to questions concerning obstacles to implementation and priorities for development. Data were organised thematically into categories, and counts of references to a particular issue or aspect of operation are reported.

\section{Results}

Of the 243 identified teams, $73 \%$ provided usable data ( $n=177) ; 54.5 \%$ described their locality as urban, 9.6\% as rural and $36 \%$ as mixed or suburban. The median team age was 20 months, heavily skewed towards younger teams; $32 \%$ of teams had been admitting service users for a year or less. Urban teams tended to be older (Kruskal-Wallis $\chi^{2}=9.01$, d.f. $=2, P=0.01$; urban $>$ suburban on multiple comparisons).

\section{Team composition}

Comparisons between reported staffing levels and projections based on the Department of Health's guidelines (2002) revealed that the number of staff working in CRHT teams was at around $88 \%$ of the recommended staffing capacity. The relationship to team maturity was complex, with younger teams (less than 2 years old) often having more capacity than their more mature counterparts.

All teams had input from nurses and most had support workers, but less than half had input from any of the other disciplines (Table 1).

\section{Case-load}

The mean case-load was 20 service users, the lower end of the policy guidance recommended range. The teams' case-loads were a mean of $59 \%$ of the recommended size taking into account the local populations. Age and size of teams were only moderate predictors of case-load size $(r=0.28, P<0.0005 ; r=0.24, P<0.001)$.

\section{Client group}

All teams accepted individuals diagnosed with psychosis or affective disorder, $84 \%$ of teams accepted those with a diagnosis of personality disorder and $42 \%$ those with a diagnosis of substance misuse.

\section{Progress towards implementation}

A measure of 'fidelity to model' was developed by determining the extent to which teams' activities fulfilled the six criteria derived from policy guidance and expert advice. The criteria and frequencies of compliance among the 150 teams that answered all six questions are given in Table 2. Teams reported meeting a mean of 4.9 of the 6 criteria.

Fidelity scale ratings were significantly higher among the $40 \%$ of teams ( $n=70$ ) who were described by their team as being fully set up to meet the needs of the numbers of people in their patch who fulfil the criteria for CRHT as defined in the Department of Health's guideline (2002), than among those that did not (mean 5.3 v. 3.9, $P<0.0001)$. original papers 
Fidelity to model was higher among urban teams (Kruskal-Wallis $\chi^{2}=9.44$, d.f. $=2, P=0.01$; urban $>$ suburban). Half of the urban teams considered themselves fully set up compared with a quarter of suburban teams and $38 \%$ of rural teams, and home visiting, telephone support and out-of-hours access were more usual in urban locations.

\section{Key operational features}

Only $43 \%$ of service users were taken on for ongoing work (median $=40 \%, n=136$ ); $22 \%$ were deemed inappropriate, and just over a third were merely assessed. Service users admitted to teams operational for less than 2 years were more likely to be referred on and less likely to be taken on for ongoing work than teams aged 2 years or more suggesting a positive impact of maturation on how teams are used. These differences were accentuated when team size was taken into account.

The most widely and intensively provided interventions beyond assessment were risk management, monitoring of mental state, assistance with self-help strategies, delivering psychosocial interventions and administering medication.

Service users came from community mental health teams (CMHT; $71 \%$ reporting daily or more frequently), accident and emergency departments (47.3\%), primary care and in-patient services (35.3\%).

Service users moved on to CMHTs (31\% daily or more often), primary care (20.9\%), voluntary sector (8.6\%) and in-patient services (8.5\%). Almost all (93\%) respondents reported delays in referral on to the local CMHT when the crisis had resolved.

\section{Threats to continued effectiveness}

Respondents were asked to describe the top three major threats to their continued effectiveness. The most frequent references were to the lack of resources to meet the demands of out-of-hours working and assessments. More staff $(n=129)$ and particularly medical input $(n=38)$ were the most widely sought after resources. Wider funding issues formed the next major category $(n=82)$. There were 67 references to inter-team problems, particularly with $\mathrm{CMHT}$ teams $(n=24)$. Medical culture, practices or attitudes formed the fourth major category $(n=55)$. This particularly concerned medical attitudes or practices that undermined the CRHT team's gate-keeping role.

\section{Perceived priorities for development}

When asked about the most useful developments or actions that would improve the effectiveness of their

\section{Table 2. Fidelity to model criteria}

Criterion

1. The team aims to provide an alternative to hospital admission for those experiencing acute mental health difficulties

2. The team stays intensively involved for as long as necessary for the immediate crisis to be resolved

3. The team acts as the gatekeeper to the acute in-patient beds by assessing people referred

4. The team is available on call or on duty between $10 \mathrm{pm}$ and $8 \mathrm{am}$

5. The team provides a 7-day per week, 24-h telephone support service

6. The team provides a 7-day per week, 24-h home-visiting assessment service 
service, team developments $(n=208)$ in the form of more staff ( $n=86)$, increased medical input $(n=30)$, and particularly dedicated consultant cover $(n=18)$ were most often cited. There were 86 references to wider improvements in local crisis services, including the need for alternative responses to crisis such as crisis beds $(n=24)$ and crisis houses $(n=18)$. There were 19 references to a need for better locally coordinated crisis response, with frequent references to the need for senior managerial support of the CHRT teams' gate-keeping role. Other ideas included better developed pathways, protocols and criteria (including the recruitment of a pathways development worker) and a manager for the emergency service covering out-of-hours crisis work.

\section{Discussion}

Although there were many well-functioning CRHT teams achieving local impact, implementation was variable. Fidelity to policy guidance was compromised in that around a third of teams were not involved in gatekeeping, and only just over half offered a 24-h, 7-day per week home visiting service. More multidisciplinary staffing was the key priority for CRHT team development. Urban teams formed the majority of CRHT teams, operated with greater fidelity to the guidance and took on a larger proportion of referrals for ongoing work. This lends fuel to the debate over whether CRHT teams offer an essentially urban solution. However, given the wider contextual factors referred to and the constraints of low staffing it would be premature to rush to this conclusion, especially that teams appear to become more targeted in their operation as they mature.

Teams were seeing fewer service users than anticipated. This may reflect lack of staff or the need to remain targeted in the face of pressures to broaden the role of the team in a way that would compromise their capacity for home treatment.

Crisis resolution/home treatment teams exist within complex local systems wherein other key resources such as CMHTs, crisis beds/houses, in-patient and primary care play key roles. Lack of support for CRHT teams in their gate-keeping role was a recurring theme, and the part played by senior medical staff in this is explored further in the accompanying paper.
Comprehensive and detailed implementation guidance does not guarantee adequate implementation even in the context of strong performance management at both provider and commissioner level. Committed and transparent funding, sufficient development support, and strong local leadership to improve inter-team working within a local whole-system of provision are also needed.

\section{Declaration of interest}

Funded through the Department of Health's Policy Research Programme.

\section{References}

DEAN, C., PHILLIPS, J., GADD, E., et al (1993) Comparison of a communitybased service with a hospital-based service for people with acute, severe psychiatric illness. BMJ, 307, 473-476. DEPARTMENT OF HEALTH (2000) The NHS Plan. HMSO

\section{DEPARTMENT OF HEALTH (2002)}

Mental Health Policy Implementation Guide: Community Mental Health Teams. Department of Health.

GLOVER, G., ARTS, G. \& BABU, K. S. (2006) Crisis resolution/home treatment teams and psychiatric admission rates in England. British Journal of Psychiatry, 189 441-445.

GLOVER, G. \& JOHNSON, S. (2008) The crisis resolution team model: recent developments and dissemination. In Crisis Resolution and HomeTreatment in Mental Health (eds S. Johnson, J. Needle, J. P. Bindman, et al). Cambridge University Press.

JOHNSON, S., NOLAN, F., HOULT, J., et al (2005) Outcomes of crisis before and after introduction of a crisis resolution team. British Journal of Psychiatry, 187, $68-75$.

JOY, C. B., ADAMS, C. E. \& RICE, K. (2006) Crisis intervention for people with severe mental illnesses. Cochrane Database of Systematic Reviews, $\mathbf{4}$, CD001087.

MIDDLETON, H., GLOVER, G., ONYETT, S., et al (2008) Crisis resolution/home treatment teams, gate-keeping and the role of the consultant psychiatrist. Psychiatric Bulletin, 32, 378-383.

MINGHELLA, E. (1998) Home-based emergency treatment. Mental Health Practice, 2, 10-14
*Steve Onyett Senior Development Consultant/Visiting Professor, Care Services Improvement Partnership South West Development Centre, Mallard Court, Express Park, Bristol Road, Bridgwater, SomersetTA6 4RN, email: steve.onyett@nimhesw.nhs.uk, Karen Linde Senior Research Fellow, Institute of Public Policy, Leeds University, Gyles Glover Consultant in Public Health, North East Public Health Observatory, and Honorary Professor of Public Mental Health, Wolfson Research Institute, University of Durham, Siobhan Floyd Research Associate, Avon and Wiltshire Mental Health Partnership NHS Trust, Steven Bradley IT Consultant, Department of Computer Science, University of Durham, Hugh Middleton Associate Professor, School of Sociology \& Social Policy, University of Nottingham, and Honorary Consultant Psychiatrist, Nottinghamshire Healthcare NHS Trust 